\begin{tabular}{|c|c|}
\hline Title & Hyper-Raman scattering enhanced by anisotropic dimer plasmons on artificial nanostructures \\
\hline Author(s) & Ikeda, Katsuy oshi; Takase, Mai; Sawai, Y oshitaka; Nabika, Hideki; Murakoshi, Kei; U osaki, Kohei \\
\hline Citation & $\begin{array}{l}\text { Journal of Chemical Physics, } 127(11), 111103 \\
\text { https://doi.org/10.1063/1.2786982 }\end{array}$ \\
\hline Issue Date & 2007-09-21 \\
\hline DOC URL & http:/hdl.handle.net/2115/50216 \\
\hline Rights & $\begin{array}{l}\text { Copyright } 2007 \text { A merican Institute of Physics. This article may be downloaded for personal use only. Any other use } \\
\text { requires prior permission of the author and the American Institute of Physics. The following article appeared in J. } \\
\text { Chem. Phys. 127, } 111103 \text { (2007) and may be found at https://dx.doi.org/10.1063/1.2786982 }\end{array}$ \\
\hline Type & article \\
\hline File Information & JCP127-11_111103.pdf \\
\hline
\end{tabular}

Instructions for use 


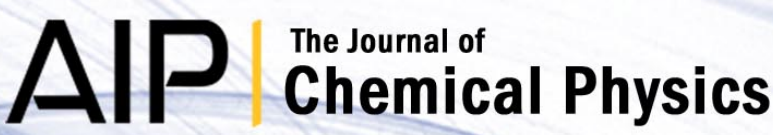

Hyper-Raman scattering enhanced by anisotropic dimer plasmons on artificial nanostructures

Katsuyoshi Ikeda, Mai Takase, Yoshitaka Sawai, Hideki Nabika, Kei Murakoshi et al.

Citation: J. Chem. Phys. 127, 111103 (2007); doi: 10.1063/1.2786982

View online: http://dx.doi.org/10.1063/1.2786982

View Table of Contents: http://jcp.aip.org/resource/1/JCPSA6/v127/i11

Published by the American Institute of Physics.

Additional information on J. Chem. Phys.

Journal Homepage: http://jcp.aip.org/

Journal Information: http://jcp.aip.org/about/about_the_journal

Top downloads: http://jcp.aip.org/features/most_downloaded

Information for Authors: http://jcp.aip.org/authors

\section{ADVERTISEMENT}

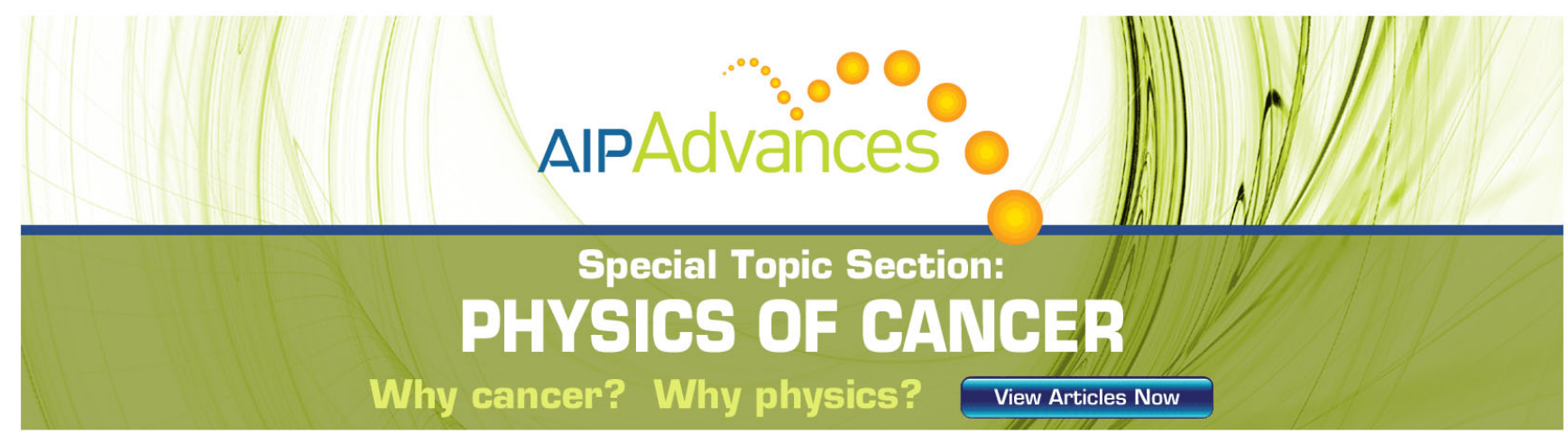




\title{
Hyper-Raman scattering enhanced by anisotropic dimer plasmons on artificial nanostructures
}

\author{
Katsuyoshi Ikeda, ${ }^{\text {a) }}$ Mai Takase, Yoshitaka Sawai, Hideki Nabika, \\ Kei Murakoshi, and Kohei Uosaki \\ Division of Chemistry, Graduate School of Science, Hokkaido University, Sapporo 060-0810, Japan
}

(Received 17 April 2007; accepted 28 August 2007; published online 18 September 2007)

\begin{abstract}
Silver nanodimers with a small gap of a few nanometers aligned on glass substrates were used to enhance hyper-Raman scattering of crystal violet dye molecules. When localized surface plasmon of the dimer array was resonantly excited along the interparticle axis, hyper-Raman intensity was significantly enhanced. Moreover, the spectral appearance was slightly different between the two excitation polarizations, suggesting a possibility of two resonance contributions at one-photon and two-photon energies. Since the plasmonic property of dimer arrays can be controlled by the dimer geometry, the dimer arrays are expected to be well-defined substrates for surface-enhanced hyper-Raman spectroscopy. (C) 2007 American Institute of Physics. [DOI: 10.1063/1.2786982]
\end{abstract}

The plasmonic properties of metallic nanostructures are attracting much attention from both fundamental and applied viewpoints. ${ }^{1-7}$ When resonantly excited, localized surface plasmon can induce giant electromagnetic (EM) field enhancement, resulting in drastic enhancement of optical scattering cross section. For example, it has been reported that the enhancement factor of surface-enhanced Raman scattering (SERS) can be more than six orders of magnitude. ${ }^{1-3}$ Such a huge effect has opened up a possibility of single molecule spectroscopy. ${ }^{4-6}$ Roughened metal substrates or metal colloid aggregates are known to exhibit huge enhancement. In these systems, however, so-called "hot" spots are found only incidentally because the enhancement factor strongly depends on their metallic nanostructures. Hence, exploration and fabrication of well-defined hot spots are currently two of the most important issues.

When two metallic nanoparticles, which nearly touch each other with a small gap of a few nanometers, are excited by polarized light parallel to the interparticle axis, the nanoparticle plasmons hybridize each other via interparticle dipole-dipole coupling, resulting in the appearance of enormous EM field around the gap. ${ }^{71}$ Since the magnitude of EM enhancement and the resonance wavelength are controllable with the dimer geometry, metallic dimers are considered as a good candidate of well-defined hot spots. Indeed, we have already reported that the metallic nanodimer arrays, which were fabricated with an angle-resolved nanosphere lithography (AR-NSL) technique, ${ }^{12}$ exhibited anisotropic and enormous enhancement of the SERS cross section. ${ }^{13}$

A nonlinear optical process would be much more sensitive to EM field enhancement because of the nonlinear dependence on excitation field. ${ }^{14}$ Hyper-Raman scattering (HRS), which is inelastic scattering in the second-order nonlinear optical process, is expected to be a useful spectroscopic technique for completing vibrational information; the

\footnotetext{
a) Author to whom correspondence should be addressed. Electronic mail: kikeda@pchem.scci.hokudai.ac.jp
}

selection rule of HRS is rather similar to that of infrared (IR) absorption. ${ }^{15-17}$ However, HRS has extremely weak effect with scattering cross sections on the order of $10^{-65} \mathrm{~cm}^{4} \mathrm{~s},{ }^{14}$ and hence, enhancement of HRS signals is indispensable. In a similar manner of SERS, surface-enhanced hyper-Raman scattering (SEHRS) has been reported in metal colloid or roughed electrode sytems. ${ }^{14,18-21}$ Then, fabrication of welldefined hot spots is awaited for further development of hyper-Raman spectroscopy.

Under these circumstances, this paper reports the enhancement of HRS by using the well-defined silver nanodimer arrays, which were fabricated with the AR-NSL technique. The uniaxially oriented dimers provide anisotropic plasmonic resonances depending on the excitation polarization: strongly hybridized dimer plasmons parallel to the axis and weakly interacting single-particle-like plasmons in the perpendicular direction. ${ }^{7-9,13}$ So, the focus is placed on anisotropic appearance of HRS in intensity and shape. Crystal violet, which is a trigonal conjugated cationic dye with large hyperpolarizability $\beta,{ }^{22}$ was selected as the test molecule because HRS originates from hyperpolarizability modulations by nuclear vibrations of molecules.

The silver nanodimer array was fabricated onto a cover slip by the AR-NSL technique as described previously. ${ }^{13}$ Briefly, silver nanodots were initially formed by vapor deposition on the cover slip masked with a polystyrene nanosphere array and then second nanodots were formed near the first dots by angle-resolved deposition. A typical structure of the dimer arrays, measured by an atomic force microscope (AFM) (Nanoscope-IIIa, Digital Instruments), is shown in Fig. 1(a). As expected from the structure, the extinction of the array was anisotropic [Fig. 1(b)]. The characteristic plasmonic resonance along the interparticle axis was found to be around $800 \mathrm{~nm}$. Since HRS was measured with $790 \mathrm{~nm}$ pulses in the present study, the polarized incidence parallel to the axis can excite the resonance accompanied with the enormously enhanced EM field around the gap. The excitation pulses with average power of $0.01 \mathrm{~nJ}$ pulse ${ }^{-1}$ and bandwidth 
(a)

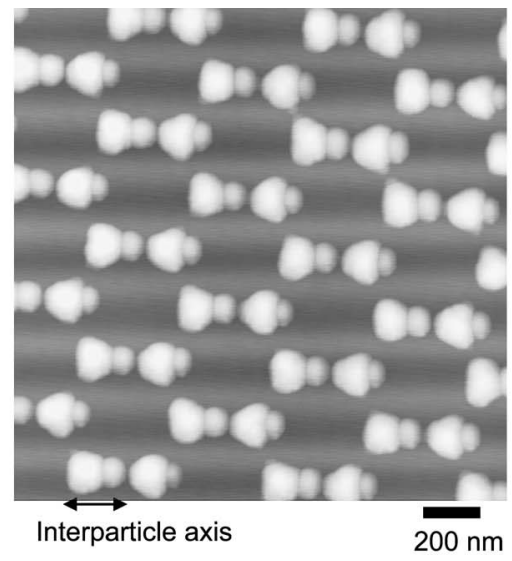

(b)

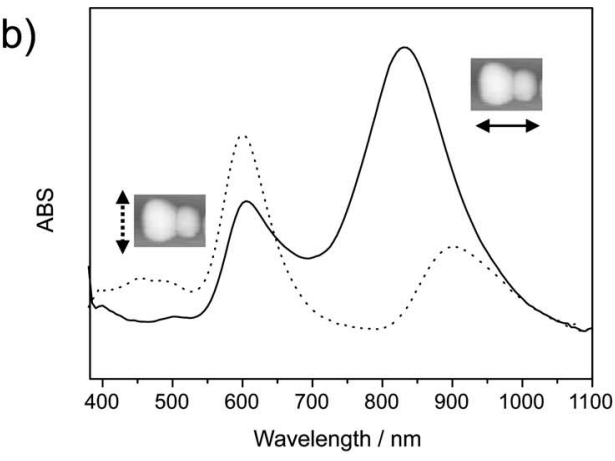

(c)

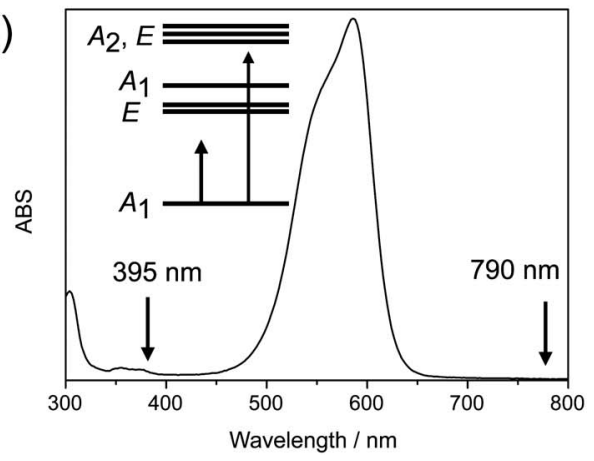

FIG. 1. (a) AFM image of the silver nanodimer array substrate fabricated with the AR-NSL technique. (b) Absorption spectra of the array parallel and perpendicular to the interparticle axis. (c) Absorption spectrum of crystal violet in methanol. Two arrows indicate the fundamental $(790 \mathrm{~nm})$ and doubled-fundamental $(395 \mathrm{~nm})$ incident energies in the SEHRS measurement. The inset diagram shows electronic ground and excited states of crystal violet.

of $14 \mathrm{~cm}^{-1}$ full width at half maximum were obtained from a mode-locked Ti:sapphire laser (Spectra-Physics, Tsunami) with a custom-made laser line filter (Optical Coatings Japan). ${ }^{23}$ An inverted microscope with a $36 \times, 0.52$ numerical aperture reflective objective was used to measure backscattered hyper-Raman signal from $0.1 \mathrm{mM}$ methanolic crystal violet solution dropped on the array. The anisotropic response of the array was measured by rotating the sample stage. In order to evaluate the degrees of contributions from one-photon and two-photon resonances, SERS spectra were also measured with two excitation wavelengths: $785 \mathrm{~nm}$ from a cw diode laser and $395 \mathrm{~nm}$ by second harmonic generation of the $790 \mathrm{~nm}$ Ti:sapphire radiation. Figure 1(c) shows absorption spectrum of crystal violet having two intense bands around $590 \mathrm{~nm}\left(S_{0} A_{1}\right.$ state $\rightarrow S_{1} E$ state $)$ and $300 \mathrm{~nm}\left(S_{0} \rightarrow S_{2} E\right.$ state) with a shoulder involving the first
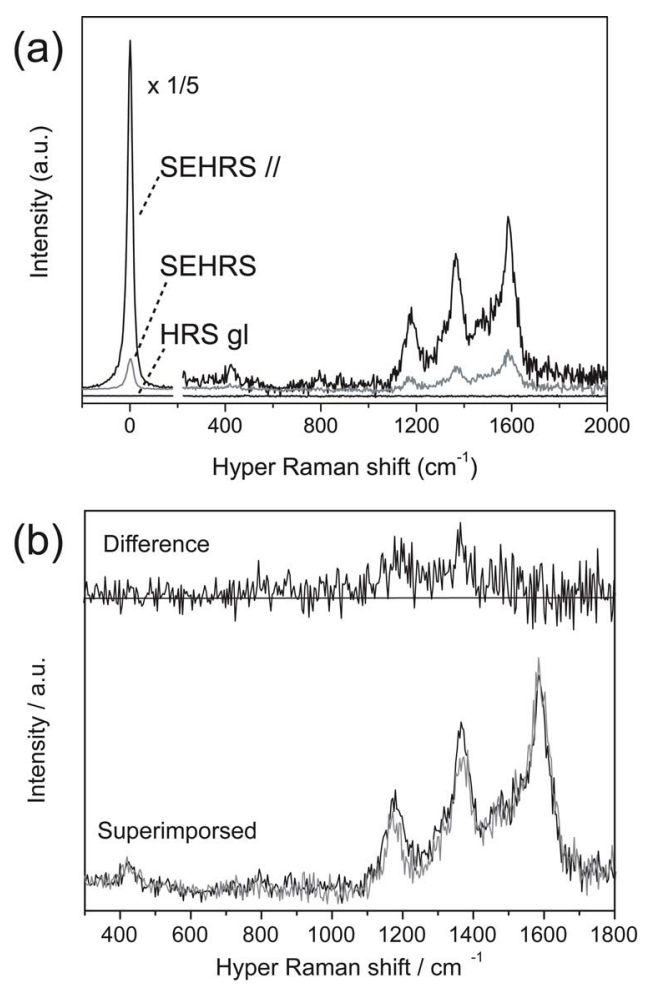

FIG. 2. (a) Three HRS spectra of crystal violet measured on glass $\left(\mathrm{HRS}_{\mathrm{gl}}\right)$ and measured on the silver dimer array with parallel (SEHRS $\|$ ) and perpendicular $(\mathrm{SEHRS} \perp$ ) excitations to the interparticle axis. Hyper-Rayleigh signal from the dimer array is scaled to one-fifth. (b) Normalized and superimposed spectra of the SEHRS $\|$ and SEHRS $\perp$ in (a) together with a difference spectrum showing that the two bands at 1177 and $1361 \mathrm{~cm}^{-1}$ were relatively weak in the case of the perpendicular excitation.

$A_{2}$ state as perturbated. ${ }^{24,25}$ The $S_{1}$ state consists of the delocalized orbitals involving a large contribution of the central carbon, whereas the $S_{2}$ state the localized benzenelike orbitals. ${ }^{26}$ The $395 \mathrm{~nm}$ excitation may be in preresonance to the latter higher band.

Figure 2(a) shows three HRS spectra of crystal violet measured on glass $\left(\mathrm{HRS}_{\mathrm{gl}}\right)$ and measured on the silver dimer array with parallel (SEHRS $\|)$ and perpendicular $($ SEHRS $\perp$ ) excitations to the interparticle axis. The HRS cross section of free crystal violet ions was so small that the $\mathrm{HRS}_{\mathrm{gl}}$ did not show any peak. On the dimer array, the cross section was substantially increased and the hyper-Raman peaks of crystal violet were clearly observed in both excitation polarizations. Especially, when excited parallel to the interparticle axis (SEHRS\|), the enhancement was much more significant. Since the local plasmons parallel to the dimer are accompanied with so-called "gap mode," gests that the gap worked as the hot spot in the HRS process. To our knowledge, this is the first demonstration of the welldefined plasmonic effect to the HRS enhancement. An estimate of the enhancement factor is difficult in this system because the hot spot size cannot be estimated accurately. However, even if the hot spot size were same as the dimer size, the enhancement factor of the dimer array would be three times larger than that of a common roughened surface. The observed three intense HRS peaks were assigned to be $\nu_{9} \mathrm{CH}$ in-plane bending at $1177 \mathrm{~cm}^{-1}, \nu(\varphi-\mathrm{N})$ stretching at $1361 \mathrm{~cm}^{-1}$, and $\nu_{8}$ ring stretching at $1584 \mathrm{~cm}^{-1}$. (The largest 
TABLE I. Principal vibrational modes of crystal violet together with frequencies and assignments (weak vibrational modes including all $A_{2}$ are omitted from the table).

\begin{tabular}{|c|c|c|c|c|c|}
\hline Symmetry & Calc. $\left(\mathrm{cm}^{-1}\right)$ & $\operatorname{Raman}\left(\mathrm{cm}^{-1}\right)$ & $\operatorname{IR}\left(\mathrm{cm}^{-1}\right)^{\mathrm{a}}$ & $\operatorname{HRS}\left(\mathrm{cm}^{-1}\right)$ & Assignment $^{\mathrm{b}}$ \\
\hline$A_{1}$ & 1615 & 1617 & & & $8 a$ \\
\hline E & 1588 & 1589 & 1584 & 1584 & \\
\hline$E$ & 1519 & 1518 & & 1522 & $8 b$ \\
\hline$A_{1}$ & 1518 & & & & \\
\hline$A_{1}$ & 1479 & 1475 & & & $19 \mathrm{a}$ \\
\hline$E$ & 1477 & & & Observed & \\
\hline$E$ & 1449 & 1446 & 1442 & Observed & $19 b$ \\
\hline$A_{1}$ & 1369 & 1376 & & & \\
\hline$E$ & 1359 & & 1362 & 1361 & $\nu(\varphi-\mathrm{N})$ \\
\hline E & 1348 & & & & \\
\hline E & 1323 & & & Observed & 14 \\
\hline$E$ & 1284 & 1296 & 1300 & Observed & \\
\hline$A_{1}$ & 1209 & 1211 & & & $9 \mathrm{a}$ \\
\hline$E$ & 1178 & 1178 & 1171 & 1177 & $9 b$ \\
\hline$A_{1}$ & 1171 & & & & \\
\hline
\end{tabular}

${ }^{\mathrm{a}}$ Taken from Ref. 23 .

${ }^{\mathrm{b}}$ Classified according to the normal modes of benzene (Ref. 23).

peak at $0 \mathrm{~cm}^{-1}$ was hyper-Rayleigh scattering from the silver dimers.) The assignments of the principal HRS modes are listed in Table I. ${ }^{26}$ The vibrational modes of free crystal violet ion with propellerlike $D_{3}$ symmetry were calculated with B3LYP/6-311G ${ }^{* *}$, which were classified into $A_{1}$ (Raman active), $A_{2}$ (IR active), and $E$ (IR/Raman active) symmetries. The three HRS peaks belong to the $E$ symmetry.

Figure 2(b) shows normalized and superimposed spectra of the SEHRS $\|$ and SEHRS $\perp$ in Fig. 2(a) together with a difference spectrum. Among the observed three HRS bands, there was an anisotropy seen in relative peak intensity; the $\nu_{9}$ and $\nu(\varphi-\mathrm{N})$ modes were less intense in the SEHRS $\perp$ than those in the SEHRS\| when normalized with the $\nu_{8}$ mode. A very similar appearance to the SEHRS $\perp$ was also observed on silver monomer arrays having a plasmonic absorption band near the doubled-fundamental incidence. Hence, one can assume that the small difference is not due to the molecular orientation. It is known that the $\nu_{9}$ and $\nu(\varphi-\mathrm{N})$ modes can couple with the $S_{0} \rightarrow S_{1}$ transition through the central carbon-phenyl displacement, and the benzenelike $\nu_{8}$ mode is enhanced by the $S_{0} \rightarrow S_{2}$ transition. ${ }^{26,27}$ Presumably, the observed difference is related to such contributions of different electronic excited states.

In order to investigate the anisotropic behavior of SEHRS on the dimer array, SERS excited by the fundamental and doubled-fundamental incident energies were compared with the SEHRS. Figure 3(a) shows the SERS spectrum excited by the $785 \mathrm{~nm} \mathrm{cw}$ polarized radiation parallel to the interparticle axis together with theoretically calculated Raman-active modes (the spectrum excited perpendicularly was not obtained because of the weak signal intensity). The observed Raman peaks are in good agreement with the calculated result; the $1617 \mathrm{~cm}^{-1}$ peak having $A_{1}$ symmetry was much more intense than the theoretically estimated intensity of the free molecule, indicating that crystal violet is attached flat on the silver surface (that is, $\mathrm{C}_{3}$ axis is normal to the surface) ${ }^{28}$ In the SERS spectra excited by the $395 \mathrm{~nm}$ pulses, on the other hand, the $\nu_{8}$ mode with $E$ symmetry was

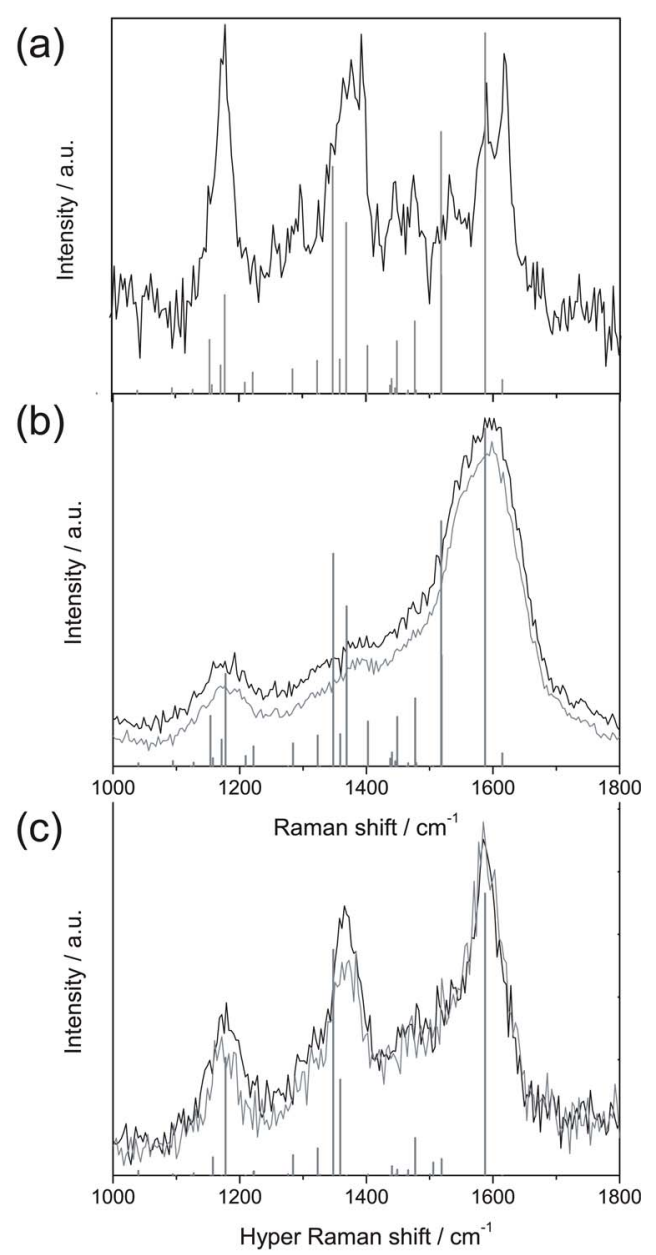

FIG. 3. SERS spectra of crystal violet onto the silver dimer arrays excited (a) by the $785 \mathrm{~nm}$ cw together with calculated Raman-active modes and (b) by the $395 \mathrm{~nm}$ pulses. (c) SEHRS spectrum excited by the $790 \mathrm{~nm}$ pulses together with calculated IR active modes. Black and gray colors represent parallel and perpendicular excitations, respectively. 
most intense as shown in Fig. 3(b). ${ }^{29}$ Note that there was no difference seen between the parallel and perpendicular excitations. This result suggests that the $\nu_{8}$ mode was, as expected from Fig. 2(b), coupled with the $S_{2}$ state. Finally, Fig. 3(c) shows the SEHRS spectrum together with the theoretically calculated IR modes. The present SEHRS should be in preresonance to the $S_{2}$ state same as the $395 \mathrm{~nm}$ excited SERS because of the noncentrosymmetry of crystal violet. In the vibronic theory for resonant hyper-Raman scattering by Chung and Ziegler et al., ${ }^{30}$ Franck-Condon-type transition dominantly contributes to the hyperpolarizability $\beta$ in a noncentrosymmetric system, meaning that resonant hyperRaman spectrum and resonant Raman spectrum excited by the doubled photon energy gain intensity through the same electronic excited state. Therefore, it is expected that the $\nu_{8}$ mode enhancement was closely related to the two-photon resonance with the $S_{2}$ state of crystal violet, while the other modes were mainly enhanced by the plasmonic resonances. If this is true, the anisotropy of the dimer plasmons can lead to the relative intensity change of these modes.

In summary, the silver nanodimer array exhibited significant enhancement of the HRS signal intensity when the hybridized dimer plasmons were resonantly excited. The degree of enhancement should be improved further by tuning the dimer geometry, e.g., the gap distance and the particle shape. Furthermore, the detailed comparison of the SEHRS spectra between the parallel and perpendicular excitations showed the difference of the relative intensity between the three intense HRS modes. As long as SEHRS is induced by conventional metal nanostructured systems with a variety of plasmonic resonances, such spectral information would disappear by averaging. The utilization of artificial nanostructures having well-defined plasmon resonances is the key to all of the information in SEHRS spectra obtained in the present study.

This research was partially supported by Grant-in-Aid for Scientific Research (A) (2006-2009, No. 18205016) and the Priority Area of "Molecular Nano Dynamics" (2004-2006, No. 16072202) from the Ministry of Education, Culture, Sports, Science and Technology, Japan. K.I. acknowledges Dr. Norihiko Hayazawa and Dr. Atsushi Ono (RIKEN, Japan) for their useful discussion.

${ }^{1}$ A. Otto, I. Mrozek, H. Grabhom, and W. Akemann, J. Phys.: Condens. Matter 4, 1143 (1992).

${ }^{2}$ F. J. Garcia-Vidal and J. B. Pendry, Phys. Rev. Lett. 77, 1163 (1996).

${ }^{3}$ Y. Yang, G. C. Schatz, and R. P. Van Duyne, J. Chem. Phys. 103, 869 (1995).

${ }^{4}$ S. M. Nie and S. R. Emery, Science 275, 1102 (1997).

${ }^{5}$ K. Kneipp, Y. Wang, H. Kneipp, L. T. Perelman, I. Itzkan, R. Dasari, and M. S. Feld, Phys. Rev. Lett. 78, 1667 (1997).

${ }^{6}$ A. M. Michaels, M. Nirmal, and L. E. Brus, J. Am. Chem. Soc. 121, 9932 (1999).

${ }^{7}$ E. Hao and G. C. Schatz, J. Chem. Phys. 120, 357 (2004).

${ }^{8}$ P. Nordlander, C. Oubre, E. Prodan, K. Li, and M. I. Stockman, Nano Lett. 4, 899 (2004)

${ }^{9}$ P. K. Aravind, A. Nitzan, and H. Metiu, Surf. Sci. 110, 189 (1981).

${ }^{10}$ P. K. Aravind and H. Metiu, J. Phys. Chem. 86, 5076 (1982).

${ }^{11}$ P. K. Aravind and H. Metiu, Surf. Sci. 124, 506 (1983).

${ }^{12}$ C. L. Haynes, A. D. McFarland, M. T. Smith, J. C. Hulteen, and R. P. Van Duyne, J. Phys. Chem. B 106, 1898 (2002).

${ }^{13}$ Y. Sawai, B. Takimoto, H. Nabika, K. Ajito, and K. Murakoshi, Faraday Discuss. 132, 179 (2006); J. Am. Chem. Soc. 129, 1658 (2007).

${ }^{14}$ J. Kneipp, H. Kneip, and K. Kneipp, Proc. Natl. Acad. Sci. U.S.A. 103, 17149 (2006)

${ }^{15}$ V. N. Denisov, B. N. Mavrin, and V. B. Podobedov, Phys. Rep. 151, 1 (1987).

${ }^{16}$ S. J. Cyvin, J. E. Rauch, and J. C. Decius, J. Chem. Phys. 43, 4083 (1965).

${ }^{17}$ J. H. Christie and D. J. Lockwood, J. Chem. Phys. 54, 1141 (1971).

${ }^{18}$ K. Kneipp, H. Kneipp, I. Itzkan, R. R. Dasari, and M. S. Feld, Chem. Phys. 247, 155 (1999).

${ }^{19}$ W.-H. Yang, J. Hulteen, G. C. Schatz, and R. P. Van Duyne, J. Chem. Phys. 104, 4313 (1996).

${ }^{20}$ W.-H. Li, X.-Y. Li, and N.-T. Yu, Chem. Phys. Lett. 327, 153 (2000)

${ }^{21}$ W. Leng, H. Y. Woo, D. Vak, G. C. Bazan, and A. M. Kelley, J. Raman Spectrosc. 37, 132 (2006).

${ }^{22}$ C. Dhenaut, I. Ledoux, I. D. W. Samuel, and J. Zyss, Nature (London) 374, 339 (1995).

${ }^{23}$ K. Ikeda, Y. Saito, N. Hayazawa, S. Kawata, and K. Uosaki, Chem. Phys. Lett. 438, 109 (2007).

${ }^{24}$ F. C. Adam and W. T. Simpson, J. Mol. Spectrosc. 3, 363 (1959).

${ }^{25}$ D. F. Duxbury, Chem. Rev. (Washington, D.C.) 93, 381 (1993).

${ }^{26}$ L. Angeloni, G. Smulevich, and M. P. Marzocchi, J. Raman Spectrosc. 8, 305 (1979).

${ }^{27}$ A. Y. Hirakawa and M. Tsuboi, Indian J. Pure Appl. Phys. 16, 176 (1978).

${ }^{28}$ Y. C. Chou, N. T. Liang, and W. S. Tse, J. Raman Spectrosc. 17, 481 (1986).

${ }^{29}$ The difference in the bandwidth has no intrinsic information because it was due to the relatively broad bandwidth of the excitation pulses.

${ }^{30}$ Y. C. Chung and L. D. Ziegler, J. Chem. Phys. 88, 7287 (1988). 